\title{
A modeled structure for amidase-03 from Bacillus anthracis
}

\author{
Ravi Datta Sharma ${ }^{1}$, Nabajyoti Goswami ${ }^{2}$, Andrew M. Lynn ${ }^{3}$, Rajnee ${ }^{4}$, Pradeep Kumar Sharma ${ }^{1}$, Safdar Jawaid ${ }^{5}$
}

${ }^{1}$ Department of Microbiology, C.C.S. University, Meerut, India; ${ }^{2}$ Center for Biotechnology, Anna University, Chennai, India; ${ }^{3}$ School of Information Technology, CCBB, Jawaharlal Nehru University, New Delhi, India; ${ }^{4}$ Department of Obstetrics and Gynecology, School of Medicine, West Virginia University, WV, USA; ${ }^{5}$ Department of Chemistry and Biochemistry, George Mason University, Fairfax, VA, USA

Received May 06, 2009; Revised October 9, 2009; Accepted October 22, 2009; Published December 31, 2009

Abstract

Homology models of amidase-03 from Bacillus anthracis were constructed using Modeller (9v2). Modeller constructs protein models using an automated approach for comparative protein structure modeling by the satisfaction of spatial restraints. A template structure of Listeria monocytogenes bacteriophage PSA endolysin PlyPSA (PDB ID: 1XOV) was selected from protein databank (PDB) using BLASTp with BLOSUM62 sequence alignment scoring matrix. We generated five models using the Modeller default routine in which initial coordinates are randomized and evaluated by pseudo-energy parameters. The protein models were validated using PROCHECK and energy minimized using the steepest descent method in GROMACS 3.2 (flexible SPC water model in cubic box of size $1 \AA$ instead of rigid SPC model). We used G43al force field in GROMACS for energy calculations and the generated structure was subsequently analyzed using the VMD software for stereo-chemistry, atomic clash and misfolding. A detailed analysis of the amidase-03 model structure from Bacillus anthracis will provide insight to the molecular design of suitable inhibitors as drug candidates.

Keywords: Homology modeling; modeller; amidase-03; hydrolase enzyme; Bacillus anthracis,

Background:

Bacillus anthracis secrets a number of enzymes capable of hydrolyzing the cell wall peptydoglycan. The hydrolysis of the cell wall imparts an important role in maintaining its structure. The structure of the cell wall therefore continuously undergoes modifications such as hydrolysis, insertion and release of old molecules $[1,2,3]$. Some enzymes, called autolysins cause cell lysis where processes like cell separation, cell wall turnover and competence occur. Autolysins also play an important role in the amplification of inflammatory responses [4]. These hydrolyzing enzymes are divided into N-acteylmuramidases, Nacetylglucosaminidases, and $\mathrm{N}$-acetylmuramoyl-L-alanine amidases based on substrate specificity [3]. The etiologic agent of anthrax, Bacillus anthracis contains two virulent plasmids $\mathrm{pXO} 1$ and pXO2 causing cutaneous, gastrointestinal and inhalation anthrax. $B$ anthracis produces protective antigen $(83 \mathrm{kDa})$, edema factor $(84$
$\mathrm{kDa})$ and lethal-factor $(83 \mathrm{kDa})$ causing inflammatory response with the release of toxic compounds and mediators [5]. The genome sequencing and annotation shows the presence of several amidases like proteins in the B. anthracis genome. The most studied amidase gene from B. anthracis is amiA, belonging to class amidase- 03 of autolysins [4]. Many autolysins usually increase the inflammatory response. However, amidase- 03 decreases the inflammatory response in host by hydrolyzing the bond between glycan and peptide thereby reducing the amplification to response [4]. So the structure stability of amidase- 03 is important to assess its function and role during infection. Therefore, it is important to construct its protein structure for amidase- 03 to glean functional information using modeling methods described elsewhere [6-8]. It should be noted that the structural analysis and molecular dynamics simulation of the predicted structure is described in detail elsewhere by the authors [9].

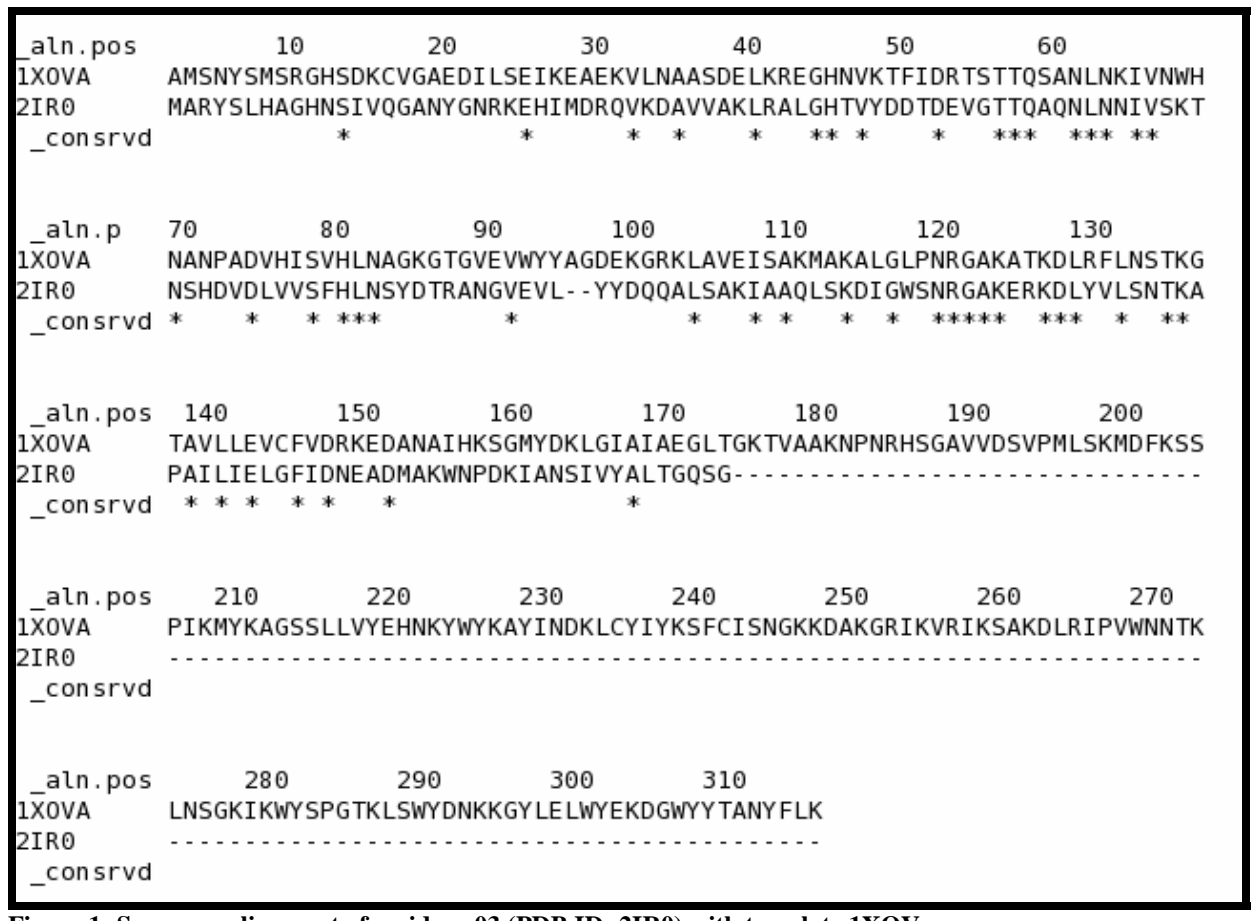

Figure 1: Sequence alignment of amidase-03 (PDB ID: 2IR0) with template 1XOV 


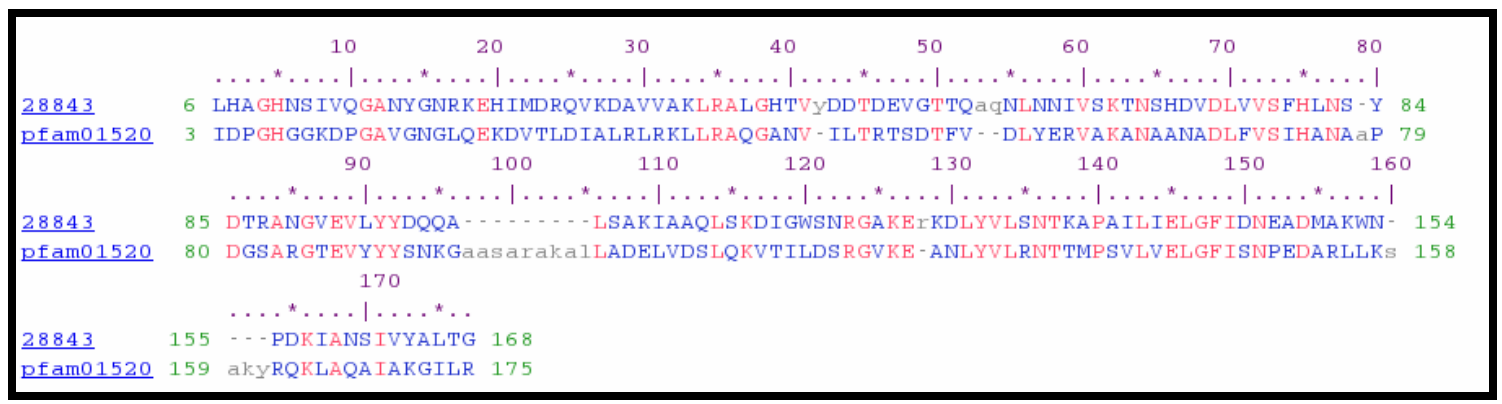

Figure 2: Sequence alignment of amidase-03 with pfam database (amidase family) as a BLASTp output

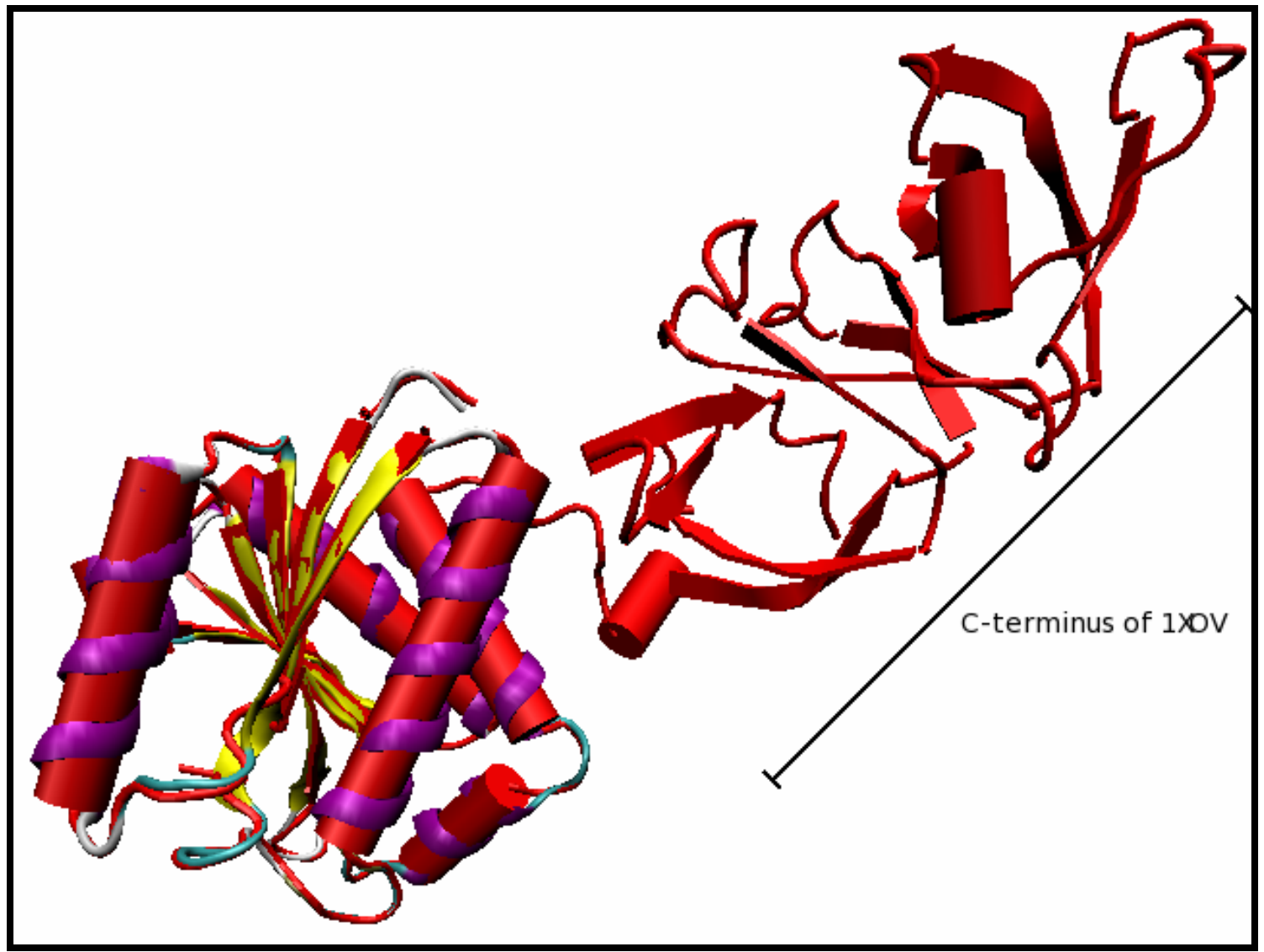

Figure 3: Superimposition of structures of amidase-03 (Red-new cartoon selection) on N-terminal of 1XOV (Ribbon selection)

Methodology:

Template Selection

Template selection was done using BLASTp for the query sequence against PDB (Protein Data Bank) available at NCBI [10]. BLOSUM62 was applied as matrix. The target subsequently selected was the x-ray crystal structure with PDB code $1 \mathrm{XOV}$ with resolution $1.8 \AA$ and R-value 0.122. Sequence alignment for template $1 \mathrm{XOV}$ and query (Figure 1) was done by using python script align2d.py in Modeller [11].

Generation of the initial structures and model evaluation Sequence alignment was used to generate several 3D models of amidase-03 using auto-model class. Five models were built by Modeller default routine in which initial coordinates are randomized and evaluated by pseudo-energy parameter. If several models are calculated for the same target, the best model can be selected in several ways. We evaluated the model with the lowest value of the
Modeller objective function and PROCHECK statistics, which is also recognized by ADIT tool at PDB Server [12]. The stereochemical properties were assessed using PROCHECK [13].

Energy minimization of the structure: Molecular Dynamics Energy minimization of the selected model was done by using steepest descent for removing the bad vander-waal contacts from the model. The calculations were done by using GROMACS 3.2 [14] software with G43a1 force field with flexible SPC water model in cubic box of size 1 Angstrom instead of rigid SPC model.

Visualization and structural analysis

Structure analysis and visualization were carried out with Visual Molecular Dynamics (VMD) [15] software. Structural alignment and RMSD calculation with template and several models were also done with VMD. 


\section{Bioinformation}

\section{Discussion:}

BLAST results had shown higher similarity with the crystal structure of $1 \mathrm{XOV}$ and $1 \mathrm{JWQ}$, while the template was selected on the basis of greater identity. 1XOV template showed $35 \%$ sequence identity, 47 conserved residues and $55 \%$ of sequence similarity with amidase-03, while the 1 JWQ showed $26 \%$ sequence identity and $55 \%$ sequence similarity. A putative domain was also found against pfam database entry pfam01520, a family of hydrolase enzyme consisting of catalytic domain which cleaves peptidoglycan of the bacterial cell wall by MurNAc-L-alanine linkage and has 51 conserved regions in BLASTp results (Figure 2). So, the 245 amino acids protein from $B$. anthracis genome encoded by GenBank accession NP 844822 consists of amidase-03 domain known to have catalytic functions to hydrolyze the cell wall. This protein in this study is referred as amidase- 03 hereon.

The template structure $1 \mathrm{XOV}$ consists of two domains, a Cterminus and $\mathrm{N}$-terminus while we used only $\mathrm{N}$ - terminus of the protein to build the model. The Figure 1 shows the alignment of 171 residues of amidase- 03 with 316 residues of $1 \mathrm{XOV}$. After 175 initial residues, Modeller put a long gap with $\mathrm{C}$-terminal of the template as we used only $\mathrm{N}$-terminus of the protein to build models of amidase-03. Due to the high target and template similarity, there are only two gaps inserted at 96th and 97th position in the $\mathrm{N}$ terminus of the sequence alignment. Using crystal structure for Homology modeling provides high quality structure alignment for structure prediction [16]. Phi and Phi torsion angles of predicted models were checked by PROCHECK. A comparative table can be found with supplementary data (Table 1). In Ramachandran plot analysis, one residue of each model was found in disallowed region. For a conformational asset, a PROCHECK analysis was done with template $1 \mathrm{XOV}$, which also confirmed one residue in disallowed region of template. So, the probable error can be negotiated here. The total number of residues in selected model was found as: $94.3 \%$ residues in most favored region, $3.8 \%$ in additional allowed region and $1.3 \%$ in generously allowed region of the Ramachandran plot.

Structural alignment was done using VMD to calculate the structure deviation between template (1XOV) and selected model of amidase03 of B. anthracis (Figure 3). The RMSD between template and selected model showed a value of $0.0976 \mathrm{~nm}$, which is an indicative of a good quality predicted model. However, all predicted models had less than $0.15 \mathrm{~nm}$ RMSD values. The predicted model of amidase- 03 consists of six $\alpha$-helices and six $\beta$-sheets. All $\beta$-sheets are surrounded with helices forming inner core of the protein and providing the amidase moiety. The overall confirmation of the model was found similar to N-terminal of 1XOV (Figure 3), which is an enzymatically active autolysin as reported in Listeria monocytogenes bacteriophage PSA endolysin PlyPSA [17]. These autolysins have unusual substrate specificity and high activity towards degradation of the murein layers and lysis of susceptible cells [17]. Based on their antimicrobial properties and substrate specificity, these enzymes show great potential to be effective therapeutic agents [18]. The increasing resistance to the known antibiotics has driven attention towards finding newer ways to combat pathogenic organisms and thus the investigation of the structures for the development of new drugs/ drug targets from the molecules like amidases have become reasonably essential especially for pathogenic organisms like B. anthracis.

\section{Conclusion}

We described the model structure for 245 amino acids amidase- 03 from Bacillus anthracis using $\mathrm{x}$-ray crystal structure of Listeria monocytogenes bacteriophage PSA endolysin PlyPSA (PDB ID: $1 \mathrm{XOV}$ ) as template. The predicted structure was submitted to Protein Data Bank (PDB-id 2IR0). Molecular dynamics simulation of the model is described elsewhere [9].

\section{Acknowledgment:}

We wish to express our sincere thanks to Professor Tasneem Fatma, Department of Bioscience, Jamia Millia Islamia, New Delhi. We also thank Dr. S. T. Pasha, National Institute of Communicable Diseases (NICD), Delhi for suggestions and support in the study.

\section{References:}

[1] AR Archibald et al., Ameri. Soci. Microbiol., 381 (1993)

[2] H Engelhardt et al., J. Struct. Biol., 124: 276 (1998) [PMID: 10049812]

[3] JM Ghuysen et al., Methods Enzymol, 8: 685 (1966)

[4] S Mesnage et al., J. Bacteriol., 1: 331 (2002) [PMCID: 134760]

[5] D Gupta et al., J. Immunol., 155: 2620 (1995) [PMID 7650392]

[6] O. Michlelin et al., J. Mol. Biol., 300: 1205 (2000)

[7] GH Paineet et al., Biopolymers, 26: 1125 (1987)

[8] R Abagyan et al., Struct. Funct. Genet., 1: 29 (1997)

[9] RD Sharma et al., Bioinformation, 3: 430 (2009)

[10] http://blast.ncbi.nlm.nih.gov/Blast

[11] MA Marti-Renom et al., Аnnu. Rev. Biophys. Biomol. Struct., 29: 291 (2000)

[12] http://deposit.pdb.org/'validate/

[13] GN Ramachandran et al., J. Mol. Biol., 7: 95 (1963) [PMID: 13990617]

[14] HJC Berendsen et al., Comp. Phys. Comm., 91: 43 (1995)

[15] W Humphrey et al., J. Mol. Graph., 14: 33 (1996) [PMID:8744570]

[16] V Chumchua et al., Bioinformation, 3: 18 (2008)

[17] IP Korndorfer et al. J. Mol. Biol., 678: 364(2006)

[18] VA Fischetti, Trends. Microbiol., 13: 491 (2005)

Edited by $P$. Kangueane

Citation: Sharma et al., Bioinformation 4(6): 242-244 (2009) License statement: This is an open-access article, which permits unrestricted use, distribution, and reproduction in any medium, for noncommercial purposes, provided the original author and source are credited. 